\title{
Levels and clinical significance of serum homocysteine (Hcy), high-density lipoprotein cholesterol (HDL-C), vaspin, and visfatin in elderly patients with different types of coronary heart disease
}

\author{
Jing Guan ${ }^{1,2 \#}$, Li Wu ${ }^{1,2 \#}$, Qian Xiao, ${ }^{1,2}$ Li Pan ${ }^{1,2}$ \\ ${ }^{1}$ Department of Geriatric ICU, Sichuan Provincial People's Hospital, University of Electronic Science and Technology of China, Chengdu, China; \\ ${ }^{2}$ Chinese Academy of Sciences, Sichuan Translational Medicine Research Hospital, Chengdu, China \\ Contributions: (I) Conception and design: J Guan, L Wu; (II) Administrative support: Q Xiao, L Pan; (III) Provision of study materials or patients: All \\ authors; (IV) Collection and assembly of data: All authors; (V) Data analysis and interpretation: All authors; (VI) Manuscript writing: All authors; (VII) \\ Final approval of manuscript: All authors. \\ \#These authors contributed equally to this work. \\ Correspondence to: Qian Xiao. Department of Geriatric ICU,32\#, West Road Section 2, First Ring Road, Chengdu 610072, China. \\ Email: evafour2011@163.com; Li Pan. Department of Geriatric ICU, 32\#, West Road Section 2, First Ring Road, Chengdu 610072, China. \\ Email: pl452932172p1@163.com.
}

Background: To investigate the levels and clinical significance of serum homocysteine (Hcy), high-density lipoprotein cholesterol (HDL-C), visceral adipose tissue-derived serine protease inhibitor (vaspin), and visceral fat-specific adipokine (visfatin) in elderly patients with different types of coronary heart disease (CHD). Methods: A total of 208 elderly patients with CHD admitted to our hospital were selected as the observation group, and 57 healthy volunteers who received physical examinations during the same period were selected as the healthy control group. The patients in the observation group were divided into a stable angina pectoris (SAP) group, an unstable angina pectoris (UAP) group, and an acute myocardial infarction (AMI) group according to their clinical diagnosis. The levels of serum Hcy, HDL-C, vaspin, visfatin, and coronary angiography Gensini scores were compared among the CHD subgroups and the healthy control group. Pearson linear correlation analysis was used to analyze the correlation between levels of serum Hcy, HDL-C, vaspin, and visfatin with Gensini scores in elderly patients with different types of CHD.

Results: The levels of serum Hcy and visfatin in the observation group were significantly higher than those in the healthy control group $(\mathrm{P}<0.05)$, while the levels of serum HDL-C and vaspin were significantly lower than those in the healthy control group $(\mathrm{P}<0.05)$. There were statistically significant differences in the levels of serum Hcy, vaspin, and visfatin among the CHD subgroups $(\mathrm{P}<0.05)$. There were statistically significant differences in the Gensini scores and number of stenotic coronary arteries among the CHD subgroups $(\mathrm{P}<0.05)$. Pearson linear correlation analysis showed that the levels of serum Hcy, HDL-C, vaspin, and visfatin in the SAP group were not significantly correlated with Gensini scores $(\mathrm{P}>0.05)$. However, the levels of serum Hcy and visfatin in the UAP and AMI groups were positively correlated with Gensini scores $(\mathrm{P}<0.05)$, the level of serum vaspin was negatively correlated with Gensini scores $(\mathrm{P}<0.05)$.

Conclusions: The levels of serum Hcy, vaspin, and visfatin vary according to the different types of CHD and are correlated with the degree of coronary artery stenosis. As such, these serum levels can be used as sensitive indicators for early detection and disease evaluation.

Keywords: Elderly coronary heart disease (elderly CHD); homocysteine (Hcy); high-density lipoprotein cholesterol (HDL-C); vaspin; visfatin; correlation

Submitted Apr 07, 2021. Accepted for publication May 23, 2021.

doi: 10.21037/apm-21-1001

View this article at: http://dx.doi.org/10.21037/apm-21-1001 


\section{Introduction}

Coronary heart disease (CHD) is a common disease in cardiovascular medicine. Coronary atherosclerosis leads to stenosis and occlusion of the vascular lumen, which causes myocardial ischemia, hypoxia, and even necrosis. Epidemiology has shown that CHD is more common in adult men over 40 years old, and its incidence increases significantly with age (1). Research reports have shown that the prevalence of CHD in patients over 60 years old can be as high as $27.8 \%$. Furthermore, in elderly patients, CHD is associated with other chronic diseases, such as hypertension and diabetes, which increase the difficulty of clinical treatment. Therefore, early diagnosis and disease evaluation are essential for improving prognosis (2). To date, some studies have shown that certain serum factors are related to the occurrence and development of CHD, but correlation analyses with disease subtypes are relatively rare (3). Homocysteine (Hcy) is an intermediate product of methionine metabolism in the body, which has the effect of damaging vascular endothelial cells and is closely related to the occurrence of cardiovascular diseases (4). High-density lipoprotein cholesterol (HDL-C) is a cholesterol receptor that can transport low-density lipoprotein cholesterol (LDL-C) to exert its anti-atherosclerotic effect (5). Visceral adipose tissue-derived serine protease inhibitor (vaspin) is a new type of adipokine, which plays an important role in maintaining the stability of the vascular environment (6). As another adipocyte factor, visfatin, promotes inflammation and aggravates atherosclerosis (7). However, the expression levels of serum Hcy, HDL-C, vaspin and visfatin in elderly patients with different types of coronary heart disease have not been studied. Accordingly, this study aims to study the levels and clinical significance of serum Hcy, HDL-C, vaspin, and visfatin in elderly patients with different types of CHD, and to provide a new reference basis for the treatment and evaluation of prognosis in elderly patients with different types of CHD. We present the following article in accordance with the STROBE reporting checklist (available at http://dx.doi.org/10.21037/apm-21-1001).

\section{Methods}

\section{General information}

A total of 208 elderly patients with CHD admitted to our hospital between January 2017 and June 2020 were selected as the observation group. All patients agreed to participate in this study and signed an informed consent form. The study was conducted in accordance with the Declaration of Helsinki (as revised in 2013). This study was approved by the Sichuan Provincial People's Hospital, University of Electronic Science and Technology of China (No. 20161216). The inclusion criteria were as follows: (I) those who met diagnostic criteria for CHD specified by the WHO in 1979 (8) and who were diagnosed by coronary angiography; (II) those who had a complete clinical examination and medical records; (III) those who had not taken drugs to improve myocardial perfusion before treatment; (IV) age $\geq 60$ years old. The exclusion criteria were as follows: (I) patients with severe arrhythmia, severe valvular heart disease, cardiogenic shock, or other clinical syndromes; (II) patients with hematological diseases, malignant tumors, systemic diseases, etc.; (III) male serum creatinine $>2.5 \mathrm{mg} / \mathrm{dL}$, female $>2.0 \mathrm{mg} / \mathrm{dL}$; (IV) liver insufficiency indicated by a liver enzyme result more than twice the normal value; (V) New York Heart Association (New York Heart Association, NYHA) grade III or IV heart failure. The duration of illness in patients in the observation group was $2-8$ years, with an average duration of $5.10 \pm 1.25$ years. In the NYHA classification, 71 cases were grade I, 89 cases were grade II, 41 cases were grade III, and 7 cases were grade IV. At the same time, 57 volunteers who received physical examinations during the same period were selected as the healthy control group. All patients with coronary artery disease were examined by coronary angiography.

\section{Studymetbod}

\section{Collection of clinical data}

General data of patients with CHD and the control group were collected on the day of examination, and all patients with CHD were followed up for 3 months. General information included name, gender, age, disease course, body mass index (BMI), years of smoking, NYHA classification, and presence of comorbid diseases. Levels of C-reaction protein (CRP), fasting plasma glucose (FPG), total cholesterol (TC), triglyceride (TG), and fasting insulin (FINS) were also measured.

\section{Index detection}

On the day of physical examination of the healthy control group and the observation group, and within 24 hours of admission, blood was collected from a vein and stored for testing. The QR-5200 model automatic biochemical analyzer (Hunan Haiyuan Medical Technology Co., Ltd., Hunan, China) was used to detect the biochemical indicators. The Hcy level was detected by the cyclic 
enzymatic method (Beijing Kangsirunye Biotechnology Co. Ltd., Beijing, China). The direct catalase removal method (Beijing Biotech Co. Ltd., Beijing, China) was used to determine the levels of HDL-C. An enzyme-linked immunosorbent assay (Shanghai Kanglang Biotechnology Co. Ltd., Shanghai, China) was used to detect the levels of vaspin. An enzyme-linked immunosorbent assay (Shanghai Jianglai Biotechnology Co. Ltd., Shanghai, China) was used to detect the level of visfatin. All detection steps and operations were carried out in accordance with the kit instructions.

\section{Degree of coronary artery stenosis}

According to the comprehensive analysis of clinical symptoms, coronary angiogram (CAG), and intravascular ultrasound results, the 208 elderly patients with CHD were divided into three groups: a stable angina pectoris (SAP) group, an unstable angina pectoris (UAP) group, and an acute myocardial infarction (AMI) group.

\section{Evaluation criteria for CHD}

The evaluation of CHD was calculated using the coronary vascular image segmentation method prescribed by the American Heart Association. The bilateral coronary arteries were observed according to the coronary angiography, and then the vessel segment was selected with a vessel diameter $\geq 2 \mathrm{~mm}$, and the stenosis position, degree, and number of vessels were recorded. The Gensini score for coronary artery disease was calculated. In this scoring system, vascular stenosis of $1-25 \%$ is rated as 1 point, $26-50 \%$ as 2 points, $51-75 \%$ as 4 points, $76-90 \%$ as 8 points, $91-99 \%$ as 16 points, and $100 \%$ as 32 points. The stenosis of different collateral vessels was observed, including the anterior descending branch, left main trunk, and circumflex branch. Among them, the proximal, middle, distal, and first diagonal branches of the anterior descending branch coefficients were rated as $\times 2.5,2,1.5$, and 1.0 , respectively; the second diagonal branch as $\times 0.5$; the left main lesion as $\times 5$; the proximal and distal circumflex branches as $\times 2.5$ or 1.0 ; the posterior descending branch and blunt marginal branch as $\times 1.0$; the right coronary artery proximal, middle, and distal parts as $\times 1.0$; the posterior descending branch as $\times 1.0$, and the left ventricular posterior collateral branch as $\times 0.5$. The Gensini score $=$ the stenosis score of each diseased coronary artery $x$ the sum of the corresponding coefficients. The degree of vascular stenosis $=($ normal blood vessel diameter near the heart of the stenosis-diameter of the stenosis/ normal blood vessel diameter near the heart of the stenosis $\times 100 \%)$. The degree of vascular stenosis is rated as follows: $\leq 50 \%$ is mild, $51-75 \%$ is moderate, $76-99 \%$ is severe, and $>99 \%$ is complete occlusion. The diagnostic criteria for CHD require that the degree of stenosis of more than one vessel is greater than $50 \%$.

\section{Observation indicators}

The clinical data of the two groups were collected, and the patients in the observation group were divided into three groups: a SAP group, an UAP group, and an AMI group based on clinical diagnosis. The levels of serum Hcy, HDL-C, vaspin, visfatin, and the coronary angiography Gensini score were compared between the different CHD subgroups and healthy controls. A Pearson linear correlation was used to analyze the correlation between the serum Hcy, HDL-C, vaspin, and visfatin levels with Gensini scores in elderly patients with different types of CHD.

\section{Statistical methods}

SPSS18.0 statistical software was used to analyze the data, and the measurement data were expressed as mean \pm standard deviation. The independent samples $t$ test was used for comparison between the two groups. One-way analysis of variance was used among multiple groups. Statistically significant results were further analyzed using the SNK-q test and Pearson linear correlations. A P value $<0.05$ was considered statistically significant.

\section{Results}

\section{Comparison of general information between the observation and control groups}

As shown in Table 1, there was no statistically significant difference between the two groups in age, gender, TG, and comorbid diseases $(\mathrm{P}>0.05)$, but the differences in BMI, smoking years, CRP, FPG, TC, and FINS were statistically significant $(\mathrm{P}<0.05)$.

\section{Comparison of levels of serum $\mathrm{Hcy}, \mathrm{HDL}-\mathrm{C}$, vaspin, and visfatin between the observation and control groups}

The levels of serum Hcy and visfatin in the observation group were significantly higher than those in the healthy control group $(\mathrm{P}<0.05)$. The levels of serum HDL-C and 
Table 1 Comparison of general data between the two groups of subjects $[\bar{x} \pm \mathrm{s}, \mathrm{n}(\%)]$

\begin{tabular}{|c|c|c|c|c|}
\hline Information & Observation group $(n=208)$ & Healthy control group $(n=57)$ & $\mathrm{t} / \chi^{2}$ value & $P$ value \\
\hline Gender & & & 0.018 & 0.893 \\
\hline Male & $137(65.87)$ & 37 (64.91) & & \\
\hline Female & $71(34.13)$ & $20(35.09)$ & & \\
\hline Smoking (years) & $16.23 \pm 3.24$ & $6.14 \pm 2.15$ & 22.195 & $<0.001$ \\
\hline $\mathrm{CRP}(\mathrm{mg} / \mathrm{L})$ & $9.12 \pm 1.24$ & $6.02 \pm 1.25$ & 16.693 & $<0.001$ \\
\hline $\mathrm{FPG}(\mathrm{mmol} / \mathrm{L})$ & $5.12 \pm 0.56$ & $4.02 \pm 0.52$ & 13.336 & $<0.001$ \\
\hline $\mathrm{TC}(\mathrm{mmol} / \mathrm{L})$ & $4.91 \pm 0.42$ & $4.01 \pm 1.12$ & 9.343 & $<0.001$ \\
\hline Comorbid diseases & & & 0.855 & 0.652 \\
\hline High blood pressure & $89(42.79)$ & $26(45.61)$ & & \\
\hline Diabetes & $47(22.60)$ & $12(21.05)$ & & \\
\hline Hyperlipidemia & $50(24.04)$ & $10(17.54)$ & & \\
\hline
\end{tabular}

BMI, body mass index; CRP, C-reaction protein; FPG, fasting plasma glucose; TC, total cholesterol; TG, triglyceride; FINS, fasting insulin; $P$ value, probability; $\chi^{2}$-test, Chi-square test.

Table 2 Comparison of levels of serum Hcy, HDL-C, vaspin, and visfatin between the two groups $(\bar{x} \pm s)$

\begin{tabular}{|c|c|c|c|c|c|}
\hline Group & $\mathrm{n}$ & Hcy (mg/L) & HDL-C (mmol/L) & Vaspin (ng/mL) & Visfatin $(\mathrm{ng} / \mathrm{mL})$ \\
\hline Healthy control group & 57 & $5.12 \pm 1.23$ & $1.49 \pm 0.23$ & $1.89 \pm 0.24$ & $10.26 \pm 2.01$ \\
\hline$t$ value & & 30.949 & 6.849 & 34.218 & 14.696 \\
\hline$P$ value & & $<0.001$ & $<0.001$ & $<0.001$ & $<0.001$ \\
\hline
\end{tabular}

Hcy, homocysteine; HDL-C, high-density lipoprotein cholesterol; P value, probability; $t$-test, Student's $t$ test.

vaspin were significantly lower than those of the healthy control group $(\mathrm{P}<0.05)$, as shown in Table 2.

\section{Comparison of levels of serum Hcy, HDL-C, vaspin, and visfatin among the CHD subgroups}

Differences in the levels of serum Hcy, vaspin, and visfatin among the CHD subgroups were statistically significant $(\mathrm{P}<0.05)$. There was no statistically significant difference in the serum HDL-C levels of the different CHD subgroups $(\mathrm{P}>0.05)$, as shown in Table 3.

\section{Comparison of the degree of coronary artery stenosis among the CHD subgroups}

The comparison of the Gensini scores and the number of stenotic coronary arteries among the CHD subgroups was statistically significant $(\mathrm{P}<0.05)$, as shown in Table 4 .

\section{Correlation between Gensini scores and the levels of serum Hcy, HDL-C, vaspin, and visfatin among the CHD subgroups}

According to the Pearson linear correlation analysis, the 
Table 3 Comparison of levels of serum Hcy, HDL-C, vaspin, and visfatin among coronary heart disease subgroups $(\bar{x} \pm \mathrm{s})$

\begin{tabular}{lccccc}
\hline Group & $\mathrm{n}$ & Hcy $(\mathrm{mg} / \mathrm{L})$ & HDL-C $(\mathrm{mmol} / \mathrm{L})$ & Vaspin $(\mathrm{ng} / \mathrm{mL})$ & Visfatin $(\mathrm{ng} / \mathrm{mL})$ \\
\hline SAP group & 92 & $12.34 \pm 2.58$ & $1.26 \pm 0.27$ & $1.23 \pm 0.24$ & $12.03 \pm 2.24$ \\
UAP group & 70 & $17.36 \pm 2.47^{\mathrm{a}}$ & $1.22 \pm 0.24$ & $0.62 \pm 0.17^{\mathrm{a}}$ & $16.35 \pm 2.37^{\mathrm{a}}$ \\
AMI group & 46 & $24.47 \pm 3.41^{\mathrm{ab}}$ & $1.20 \pm 0.26$ & $0.30 \pm 0.12^{\mathrm{ab}}$ & $19.89 \pm 2.40^{\mathrm{ab}}$ \\
F value & & 301.325 & 0.968 & 399.191 & $<8.470$ \\
P value & & $<0.001$ & 0.381 & $<0.001$ & $<0.001$ \\
\hline
\end{tabular}

Compared with SAP group, ${ }^{a} \mathrm{P}<0.05$; compared with UAP group, ${ }^{b} \mathrm{P}<0.05$. Hcy, homocysteine; HDL-C, high-density lipoprotein cholesterol; SAP, stable angina pectoris; UAP, unstable angina pectoris; AMI, acute myocardial infarction; $\mathrm{P}$ value, probability; F value, oscillator strength.

Table 4 Comparison of degree of coronary artery stenosis among coronary heart disease subgroups $[\bar{x} \pm \mathrm{s}, \mathrm{n}(\%)]$

\begin{tabular}{|c|c|c|c|}
\hline Group & $\mathrm{n}$ & Coronary angiography Gensini score & Number of stenotic coronary arteries \\
\hline UAP group & 70 & $35.23 \pm 9.35^{a}$ & $1.96 \pm 0.35^{\mathrm{a}}$ \\
\hline AMI group & 46 & $59.25 \pm 14.02^{\mathrm{ab}}$ & $2.67 \pm 0.51^{\mathrm{ab}}$ \\
\hline F value & & 438.051 & 768.919 \\
\hline
\end{tabular}

Compared with SAP group, ${ }^{\mathrm{a}} \mathrm{P}<0.05$; compared with UAP group, ${ }^{\mathrm{b}} \mathrm{P}<0.05$.SAP, stable angina pectoris; UAP, unstable angina pectoris; AMI, acute myocardial infarction; $P$ value, probability; $F$ value, oscillator strength.

Table 5 Correlation between levels of serum Hcy, HDL-C, vaspin and visfatin with coronary angiography Gensini score among coronary heart disease subtypes

\begin{tabular}{|c|c|c|c|c|}
\hline Group & Hcy & HDL-C & Vaspin & Visfatin \\
\hline \multicolumn{5}{|l|}{ SAP group } \\
\hline rvalue & 0.196 & 0.123 & 0.110 & 0.189 \\
\hline$P$ value & $>0.05$ & $>0.05$ & $>0.05$ & $>0.05$ \\
\hline \multicolumn{5}{|l|}{ UAP group } \\
\hline $\mathrm{P}$ value & $<0.05$ & $>0.05$ & $<0.05$ & $<0.05$ \\
\hline \multicolumn{5}{|l|}{ AMI group } \\
\hline rvalue & 0.528 & 0.204 & -0.427 & 0.508 \\
\hline$P$ value & $<0.05$ & $>0.05$ & $<0.05$ & $<0.05$ \\
\hline
\end{tabular}

Hcy, homocysteine; HDL-C, high-density lipoprotein cholesterol; SAP, stable angina pectoris; UAP, unstable angina pectoris; AMI, acute myocardial infarction; $\mathrm{P}$ value, probability; $r$ value, right value.

serum Hcy, HDL-C, vaspin, and visfatin levels in the SAP group were not significantly correlated with Gensini scores $(\mathrm{P}>0.05)$. Serum Hcy and visfatin levels in the UAP group and AMI group were positively correlated with Gensini scores $(\mathrm{P}<0.05)$ and the serum vaspin level was negatively correlated with Gensini scores $(\mathrm{P}<0.05)$. There was no significant correlation between the serum HDL-C level and Gensini scores $(\mathrm{P}>0.05)$, as shown in Table 5 . 


\section{Discussion}

In recent years, with the increasing mortality associated with CHD in the elderly, clinical exploration and prevention of CHD risk factors have received extensive attention worldwide (9). Based on this, strengthening the research of new biomarkers is the key to early screening and diagnosis and to improving the effectiveness of treatment. The pathogenesis of CHD involves onset of arterial thrombosis and atherosclerosis, which lead to myocardial ischemia, hypoxia, and arterial stenosis. The risk factors may be related to factors such as smoking, obesity, age, and family genetic history. At present, the relationship between new risk factors related to hyperhomocysteinemia, adipokines, and dyslipidemia, and the incidence and progression of CHD in the elderly is gradually receiving more attention (10). The results of this study showed that compared with healthy controls of the same age, elderly patients with CHD had statistically significant differences in BMI, smoking years, CRP, FPG, TC, and FINS. This is similar to a previous report on the risk factors of CHD in the elderly (11). This is mainly because CHD is a disease caused by multiple factors and believed to be mainly related to dyslipidemia (12). In recent years, some studies have confirmed a relationship between inflammation and risk factors for CHD (13). CRP levels are regulated by inflammatory factors secreted by visceral adipocytes, which in turn participate in lipid metabolism disorders and appear to increase insulin resistance and blood pressure. For this reason, early clinical screening of high-risk groups with relevant risk factors can effectively reduce the incidence of CHD.

At present, there are many clinical studies on the risk factors of CHD in the elderly, and it has been confirmed that many serum cytokines are involved in the pathogenesis and progression of CHD. However, the risk factors between different types of CHD and the relationship with related serum indicators are less well-known (14). Angina pectoris and myocardial infarction are the two main types of CHD, and the former can be simply divided into SAP and UAP according to the World Health Organization naming and diagnostic criteria of ischemic heart disease. SAP's pathological symptoms usually appear during physical activity, and the nature of the disease is stable and unchanged within a 1 - to 3 -month period. The daily pain time, location, and inducement are roughly the same, and it can be relieved within a relatively short period of time after using nitroglycerin. UAP lies between SAP and AMI, and includes initial exertional angina, resting angina, and post- infarction angina. In regard to coronary atherosclerosis, any lack of myocardial blood supply and oxygen supply can cause the disease. In regard to SAP, the condition can suddenly worsen and show an increase in the number of attacks, prolonged pain time, and weakened nitroglycerin treatment effect. If not treated in time, coronary artery constriction and stenosis can lead to severe insufficiency of blood supply to the myocardium, resulting in myocardial necrosis, which develops into the clinical syndrome of AMI (15).

This study explored the comparison of the abovementioned different types of CHD in elderly patients with CHD-related serum indicators. The results showed that there were significant differences in the serum Hcy, vaspin, and visfatin levels between the different CHD subgroups, suggesting that early detection of the above index levels can be used to identify clinical SAP, UAP, and AMI to a certain extent. The levels of serum HDL-C were equivalent, which indicates that although HDL-C is involved in the pathogenesis of CHD in the elderly, it has no obvious significance for the classification and identification of CHD. In addition, the comparison of Gensini scores and the number of stenotic coronary arteries among the three groups was statistically significant, confirming that the pathological development from SAP to UAP to AMI has a certain pattern. The coronary artery disease of patients with UAP and AMI is so severe that it affects their condition. Correlation analysis showed that serum Hcy and visfatin levels in the UAP group and AMI group were positively correlated with the coronary angiography Gensini score, and the serum vaspin level was negatively correlated with the coronary angiography Gensini score. The fundamental reason is that Hcy is an intermediate product of methionine metabolism, which is a type of neurotransmitter. Under normal physiological conditions, its release and metabolism maintain a balanced state, and when the body develops disease, its level will also change (16). Elderly patients with CHD have abnormally increased Hcy levels due to changes in their eating habits and internal environment, which promote the release of superoxide and inhibit the production of antioxidant enzymes. In turn, this causes a strong oxidative stress response in the body and damages endothelial cells. The damage of endothelial cell function can lead to an imbalance in the level of active substances in the blood vessels, resulting in arterial spasm and plaque splitting. Similarly, the decline of the anti-blood activity of endothelial cells can increase thrombosis, and eventually cause aggravation of the disease. Previous studies have suggested that the reduction of HDL-C levels and the increase of LDL-C are independent risk factors for the onset 
of CHD (17). Holmes et al. (18) pointed out that low-level HDL-C is associated with a higher incidence of coronary artery disease. The results of the current study indicate that the level of serum HDL-C can be used as an independent indicator for the clinical diagnosis of CHD in the elderly. But it cannot be used for the identification of different clinical subtypes. The above results are inconsistent, which may be related to sample size, inclusion criteria, reagent sensitivity, the distribution of patient conditions, or other factors affecting the number of coronary artery lesions and different types. Vaspin is a recently discovered adipokine, which can reduce the release of inflammatory factors such as CRP by inhibiting the adhesion of monocytes in the body thus reducing the degree of inflammation. As the stability of coronary plaques decreases, myocardial ischemia intensifies and increases the risk of myocardial infarction, and the substances reflecting myocardial damage increase significantly. Studies have found that vaspin levels are significantly negatively correlated with myocardial injury markers such as creatine kinase isoenzymes, which in turn participate in the occurrence and progression of CHD (19). Visfatin is not only a fat factor but also an important proinflammatory factor in the body. It increases the release of interleukin-6 and other inflammatory factors by inducing the activation of transcription factors. In addition, studies have shown that visfatin can also promote the proliferation and degeneration of vascular smooth muscle cells, thereby causing vascular endothelial cell function damage, which promotes the formation of atherosclerosis (20).

The disadvantage of this study is that it is an observational study. Therefore, the existence of confounding bias is inevitable, and it is difficult to fully uncover the relationship between the different subtypes of CHD in elderly patients. In addition, there are certain limitations to the study in regard to regional restrictions for sample inclusion, and a lengthy time span. These issues will be addressed in our future research.

In summary, there are changes in serum Hcy, HDL-C, vaspin, and visfatin levels in elderly patients with CHD. Among them, the levels of Hcy, vaspin, and visfatin can be used as clinical indicators to distinguish SAP, UAP, and AMI, and are negatively correlated with coronary angiography Gensini scores. Early detection is helpful for diagnostic screening and disease evaluation.

\section{Acknowledgments}

Funding: None.

\section{Footnote}

Reporting Checklist: The authors have completed the STROBE reporting checklist. Available at http://dx.doi. org/10.21037/apm-21-1001

Data Sharing Statement: Available at http://dx.doi. org/10.21037/apm-21-1001

Conflicts of Interest: All authors have completed the ICMJE uniform disclosure form (available at http://dx.doi. org/10.21037/apm-21-1001). The authors have no conflicts of interest to declare.

Etbical Statement: The authors are accountable for all aspects of the work in ensuring that questions related to the accuracy or integrity of any part of the work are appropriately investigated and resolved. All patients agreed to participate in this study and signed an informed consent form. The study was conducted in accordance with the Declaration of Helsinki (as revised in 2013). This study was approved by the Sichuan Provincial People's Hospital, University of Electronic Science and Technology of China (No. 20161216).

Open Access Statement: This is an Open Access article distributed in accordance with the Creative Commons Attribution-NonCommercial-NoDerivs 4.0 International License (CC BY-NC-ND 4.0), which permits the noncommercial replication and distribution of the article with the strict proviso that no changes or edits are made and the original work is properly cited (including links to both the formal publication through the relevant DOI and the license). See: https://creativecommons.org/licenses/by-nc-nd/4.0/.

\section{References}

1. Wirtz PH, von Känel R. Psychological Stress, Inflammation, and Coronary Heart Disease. Curr Cardiol Rep 2017;19:111.

2. Sun H, Li XN, Zhang AH, et al. Exploring potential biomarkers of coronary heart disease treated by Jing Zhi Guan Xin Pian using high-throughput metabolomics. RSC Advances 2019;9:11420-32.

3. Wang S, Xiu C, Su Y, et al. GW29-e0361 Clinical value of myocardial contrast perfusion to detect coronary heart disease during stress-echocardiography. J Am Coll Cardiol2018;72:198-9. 
4. Li S, Pan G, Chen H, et al. Determination of Serum Homocysteine and Hypersensitive C-reactive Protein and Their Correlation with Premature Coronary Heart Disease. Heart Surg Forum 2019;22:E215-7.

5. Li Z, Huang J, Li N. Predictive and Prognostic Value of High-density Lipoprotein Cholesterol in Young Male Patients with Acute Myocardial Infarction. Chin Med J (Engl) 2017;130:77-82.

6. Suliga E, Kozieł D, Cieśla E, et al. Associations Between Vaspin Rs2236242 Gene Polymorphism, Walking Time and the Risk of Metabolic Syndrome. Balkan J Med Genet 2019;22:41-8.

7. Rodrigo C, Tennekoon KH, Karunanayake EH, et al. Circulating leptin, soluble leptin receptor, free leptin index, visfatin and selected leptin and leptin receptor gene polymorphisms in sporadic breast cancer. Endocr J 2017;64:393-401.

8. Cohn PF. Asymptomatic coronary artery disease. Pathophysiology, diagnosis, management. Mod Concepts Cardiovasc Dis 1981;50:55-60.

9. Abaci O, Kocas C, Oktay V, et al. Relationship between myocardial performance index and severity of coronary artery disease in patients with non-ST-segment elevation acute coronary syndrome. Cardiovasc J Afr2017;28:4-7.

10. Sun D, Wu Y, Wang H, et al. Toll-like receptor 4 rs11536889 is associated with angiographic extent and severity of coronary artery disease in a Chinese population. Oncotarget2017;8:2025-33.

11. Farnier M, Salignon-Vernay C, Yao H, et al. Prevalence, risk factor burden, and severity of coronary artery disease in patients with heterozygous familial hypercholesterolemia hospitalized for an acute myocardial infarction: Data from the French RICO survey. J Clin Lipidol2019;13:601-7.

12. Ji J, Liu Y, Liu H, et al. Relationship between Procalcitonin, Homocysteine and Severity of Coronary

Cite this article as: Guan J, Wu L, Xiao Q, Pan L. Levels and clinical significance of serum homocysteine (Hcy), high-density lipoprotein cholesterol (HDL-C), vaspin, and visfatin in elderly patients with different types of coronary heart disease. Ann Palliat Med 2021;10(5):5679-5686. doi: 10.21037/apm-21-1001
Artery Disease in Type 2 Diabetic Patients. Int J Gerontol2019;13:226-30.

13. Yan W, Song Y, Zhou L, et al. Immune Cell Repertoire and Their Mediators in Patients with Acute Myocardial Infarction or Stable Angina Pectoris. Int J Med Sci 2017;14:181-90.

14. Kumar A, Shariff M, Doshi R. Association Between Past Hepatitis B Infection and Ischemic Heart Disease: An Analysis From the 2007-2016 NHANES Data. Am J Med Sci 2020;360:372-7.

15. Lin Y, Dan H, Lu J. Overexpression of microRNA-136-3p Alleviates Myocardial Injury in Coronary Artery Disease via the Rho A/ROCK Signaling Pathway. Kidney Blood Press Res 2020;45:477-96.

16. Chen S, Luan H, He J, et al. The relationships of serum homocysteine levels and traditional lipid indicators with disease activity and coronary artery involvement in Takayasu arteritis. Immunol Res 2020;68:405-13.

17. Zafrir B, Jubran A, Lavie G, et al. Clinical Features and Gaps in the Management of Probable Familial Hypercholesterolemia and Cardiovascular Disease. Circ J 2017;82:218-23.

18. Holmes MV, Millwood IY, Kartsonaki C, et al. Lipids, Lipoproteins, and Metabolites and Risk of Myocardial Infarction and Stroke. J Am Coll Cardiol2018;71:620-32.

19. Paltrinieri S, Pintore L, Balducci F, et al. Serum creatine kinase isoenzymes and macroenzymes in dogs with different neurologic diseases. Vet Clin Pathol2017;46:91-9.

20. Ma J, Zhao X, Zhang J, et al. Aldosterone may induce visfatin expression via the GR-ERK1/2 signal pathway in 3T3-L1 adipocytes. Acta Biochim Biophys Sin (Shanghai) 2019;51:331-4.

(English Language Editor: D. Fitzgerald) 